\title{
Endogenous or Exogenous Origin of Vaginal Candidiasis in Polish Women?
}

\author{
MAGDALENA MNICHOWSKA-POLANOWSKA ${ }^{1 *}$, IWONA WOJCIECHOWSKA-KOSZKO ${ }^{1}$, \\ BOGUMIA KLIMOWICZ ${ }^{1}$, LUDMIA SZYMANIAK, BARBARA KRASNODĘBSKA-SZPONDER, \\ ZBIGNIEW SZYCH${ }^{2}$ and STEFANIA GIEDRYS-KALEMBA ${ }^{1}$ \\ ${ }^{1}$ Pomeranian Medical University, Dept of Microbiology and Immunology, Szczecin, Poland
${ }^{2}$ Pomeranian Medical University, Dept of Computer Science and Education Quality Research, Szczecin, Poland
}

Submitted 30 October 2012, revised 12 January 2013, accepted 31 January 2013

\begin{abstract}
Vaginal candidiasis is a common problem of clinical practice. Many studies have been conducted to explain its origin but only a few have included Polish women. The aim of the study was to determine the prevalence and similarity of oral, anal and vaginal Candida albicans strains isolated from Polish women with vaginal candidiasis. The study involved 20 from 37 recruited women. Swab samples were collected from their vagina, anus, and oral cavity at two-month intervals. All the women were treated with nystatin. Yeast were recovered and identified by the germ-tube test, API /Vitek system, typed by API ZYM and RAPD-PCR. Chi-square test was used to analyze the data. A total of 170 Candida albicans isolates were recovered from 180 samples collected 3 times from 3 sites of 20 women. Positive yeast vaginal cultures were found in all patients before administration of nystatin. Vaginal yeast recovery rate was decreased statistically significant in both follow-up visits $(p=0.001 ; p=0.003)$. The same and different genotypes/biotypes were found concomitantly in a few body sites and/ or repeatedly at time interval from the same body site. The results support the concept of dynamic exchange of yeast within one woman and endogenous or exogenous origin of vaginal candidiasis.
\end{abstract}

Ke y words: Candida albicans, candidiasis, molecular typing

Vaginal candidiasis (VC) is recognized in $20-50 \%$ of women attending primary care clinics because of vaginal complaints (Eschenbach, 2008; Ilkit and Guzel, 2011; Vazquez et al., 1994). Peak incidence of VC corresponds to a period of high sexual activity (20-40 years of age) and high-estrogen host status, uncommon before menarche and after menopause (Amouri et al., 2010; Lanchares and Hernández, 2000). The most important factors predisposing to $\mathrm{VC}$ are: uncontrolled hyperglycemia, pregnancy, use of highestrogen oral/intrauterine contraceptives, decreased immune response due to neoplastic diseases or immunosuppression, as well as abuse of broad-spectral antibiotics in combination with yeast vaginal colonization (Amouri et al., 2010; Sobel, 2007). Some studies have in several cases failed to find predisposing factors to VC (Sobel, 1985), thus from the clinical point of view VC is characterized as an oestrogen - related hypersensitivity response to a commensal organism (Fischer, 2012). About $75 \%$ of women have at least one episode of VC during their adult life and are treated with antifungal drugs (Hurley and DeLouvois, 1979; Lanchares and Hernández, 2000; Maffei et al., 1997; Sobel, 2007). Some of them can be culture-positive for yeast in the vagina, experience relapse (20-50\%) or progress of symptoms within 1-3 months after clinically successful therapy (Amouri et al., 2010; Lanchares and Hernández, 2000; Mercure et al., 1993; Ringdahl, 2000; Sobel, 1996; Sobel, 1990; Vazquez et al., 1994) and approximately 5\% have a recurrent infection (Sobel, 2007; Sobel, 1993).

Although infections due to non-albicans Candida species are increasing in prevalence (10-30\%), multiple research studies permanently recognize Candida (C.) albicans as the most important infectious agent of VC with prevalence to $80-95 \%$ in various reports (Amouri et al., 2010; Chong et al., 2003; Darce Bello et al., 2002; Mahmoudi Rad et al., 2011; Mendling et al., 2000; Sobel, 2007; Sobel, 1993; Sobel, 1985). C. albicans belongs also to the most common fungal species isolated from vaginas of women without clinical manifestation (al-Rawi and Kavanagh, 1999; Chong et al., 2003; Darce Bello et al., 2002; Li et al., 2008; Mendling et al., 2000; Sobel, 1985; Vazquez et al., 1994). Vaginal colonization is asymptomatic in 20\% (range 10-80\%) of women, higher in pregnancy (especially at week 28 or later) and in the women, who possess VC risk factors (Lanchares and Hernández, 2000; Mahmoudi Rad et al., 2011; Sobel, 2007).

\footnotetext{
* Corresponding author: M. Mnichowska-Polanowska, Pomeranian Medical University, Dept of Microbiology and Immunology, Powstańców Wlkp. 72 street, 70-11 Szczecin, Poland; phone: + 4891466 16 52; fax: + 4891466 16 59; e-mail: rumianek1978@wp.pl
} 
Circulation of Candida strains between the vagina and other colonized body sites can be responsible for the difficulties in treatment and management of VC. The oral cavity and anus appear to be a site of C. albicans persistence and a source of endogenous re-infection (Lanchares and Hernández, 2000; Sobel, 2007). Molecular methods used for genetic typing of strains have revealed similarity as well as diversity between oral, anal and vaginal C.albicans isolates (Ge et al., 2012; Schmid et al., 1993; Xu et al., 1999). Colonization of the vagina by isolates of the same type for a longer time period can explain why moderate symptoms of VC or relapse after treatment are observed in some women (Lanchares and Hernández, 2000; Sobel, 1985; Vazquez et al., 1994). The diversity of molecular types among strains isolated from the site of infection and colonized areas may suggest re-infection, not necessarily caused by endogenous strain, but by vaginopathic isolate derived from exogenous sources (Ge et al., 2012; Li et al., 2008). Up to now, many studies regarding VC have been conducted to explain the microbiological origin and epidemiology of VC in the world. However, only a few have included Polish women.

The objective of this work was to determine the prevalence and similarity of oral, anal and vaginal C. albicans strains isolated from women with VC in a period of 4-months with using phenotyping and genotyping methods.

Patients with VC from West Pomeranian region of Poland were recruited to the study $(n=37)$ based on a written questionnaire. Women in pregnancy, with diabetes mellitus, immunodeficiency, neoplastic diseases, receiving oral contraceptives, immunosuppressed drugs or antibiotics were excluded from the study. All signed a written consent for participation and re-attendance at follow-up visits after VC treatment. Of the women recruited 20 completed the study. They were aged 21-44 years (average 33). No known sister-sister, mother-sister, or cohabitation relationships were identified among the subjects. All the women presented symptoms of vaginal yeast infection during the first visit and received nystatin. All of them were under the care of the same gynecologist, who collected samples from the vagina, anus, and oral cavity three times at two-month intervals: I - after diagnosis of VC and before topical treatment with nystatin, II - after 2 months, III - after 4 months. Swab specimens were cultured at $37^{\circ} \mathrm{C}$ for $48 \mathrm{~h}$ on SDA (Sabouraud Dextrose Agar) medium for yeast isolation and on chromogenic agar medium for estimation of yeast morphology. All morphologically distinct yeast colonies were recorded as "different" isolates and were identified by the germ tube test, API ID $32 \mathrm{C}$ kit or by the Vitek Compact 2 system (BioMérieux SA, France). Subsequently, all strains were biotyped using API ZYM (BioMérieux SA,
France) and genotyped using optimized RAPD-PCR (random amplified polymorphic DNA). Biotypes were determined based on activity of 5 hydrolases: esterase $\mathrm{C} 4$, valine arylamidase, naphtol-AS-BI-phosphohydrolase, $\alpha$-glucosidase, $\mathrm{N}$-acetyl-beta-glucosamidase (Kurnatowska and Kurnatowski, 1998; Wiliamson et al., 1986). In optimized RAPD-PCR method was used the primers set: 1290 (5'-GTG GAT GCG A-3')/1247 (5'-AAG AGC CCG T-3') (Cobb and Clarkson, 1994; Cobb and Clarkson, 1992; Schmid et al., 1990). The genetic similarity of the isolates was calculated using the Dice similarity coefficient $\left(\mathrm{S}_{\mathrm{AB}}\right)$, clustered by the unweighted pair group method (UPGMA) and visualized by the dendrogram (Sobel, 2003; Soll et al., 1991). The threshold for clusters of genetically related and different isolates was calculated respectively at a $S_{A B} \geq 0.80$ and at a $\mathrm{S}_{\mathrm{AB}}<0.80$. The control organism used in the study was C. albicans ATCC 24433.

The occurrence of yeast-positive cultures in a single body site in the following visits was compared $\chi$-square test with Yates correction factor and Fisher's exact probability test (two side-test). A comparison of yeast-positive cultures between two or three examined body sites within a single visit performed respectively by McNemar's test and Cochrane Q test was made. Distribution of the number of $C$. albicans isolates in a single body site between visits was analyzed using comparison of 2 counts. In all calculations assumed p-values of $<0.05$ statistically significant.

A total of 139 yeast-positive cultures (77.2\%) and 170 isolates of C. albicans were recovered from $180 \mathrm{swab}$ samples collected three times from 3 body sites of 20 women. During the first sampling before treatment, positive vaginal cultures were found at all symptomatic patients. In the follow-up visits (II and III) yeast-positive samples from vagina were recovered respectively in $50 \%$ and $60 \%$ of cases.

Statistically significant differences were noted in the yeast recovery rate from vagina between visits I and II as well as between visits I and III, $p=0.001$ and $p=0.003$, respectively. Significantly less vaginal C. albicans strains isolated from visit II in comparison with the visit I $(p=0.05)$ (Table I). During one sampling C.albicans positive cultures were most commonly found in all three body sites at once $-46.7 \%$. Differences in yeast-positive samples between three studied body sites within visits I and II were statistically significant $(p=0.04)$ (Table I). The colonization of two sites occurred with $38.4 \%$, mostly in the combinations: anus - oral cavity and vagina - oral cavity. Single sites were colonized rarely, mostly oral cavity (Table II).

Mixed cultures as a combination of C. albicans and non-albicans species (C. parapsilosis or C. dubliniensis) were found at 2 women (K.I, F.U.) only but the nonalbicans strains $(\mathrm{n}=2)$ were excluded from further 
Table I

The number and percentage of yeast-positive cultures and C. albicans strains isolated from 3 body sites of women during 3 consecutive visits

\begin{tabular}{|c|c|c|c|c|c|c|c|c|c|c|}
\hline \multirow{3}{*}{$\begin{array}{l}\text { No of visits/ } \\
\text { samples }\end{array}$} & \multicolumn{7}{|c|}{ Yeast - positive cultures; n (\%) } & \multicolumn{3}{|c|}{ Candida albicans isolates; (n) } \\
\hline & \multirow{2}{*}{ vagina } & \multirow{2}{*}{ oral cavity } & \multirow{2}{*}{ anus } & \multirow{2}{*}{$\begin{array}{c}\text { p-value } \\
\text { (Cochrane } \\
\text { Q test) }\end{array}$} & \multicolumn{3}{|c|}{$\mathrm{p}$-value McNemar's test } & \multirow{2}{*}{ vagina } & \multirow{2}{*}{ oral cavity } & \multirow{2}{*}{ anus } \\
\hline & & & & & vagina-oral & vagina-anus & oral-anus & & & \\
\hline $\mathrm{I} n=20 / 20 / 20$ & $20(100)$ & $18(90)$ & $15(75)$ & 0.04 & 0.5 & 0.1 & 0.3 & 24 & 25 & 20 \\
\hline II $n=20 / 20 / 20$ & $10(50)$ & $17(85)$ & $16(80)$ & 0.04 & 0.05 & 0.1 & 1.0 & 10 & 19 & 18 \\
\hline III $n=20 / 20 / 20$ & $12(60)$ & $18(90)$ & $13(65)$ & 0.09 & 0.1 & 1.0 & 0.7 & 15 & 22 & 17 \\
\hline \multicolumn{8}{|c|}{$\begin{array}{l}\text { p-value }-\chi \text {-square test with Yates correction factor or Fisher's exact probability test } \\
\text { (two side-test) }\end{array}$} & \multicolumn{3}{|c|}{$\begin{array}{l}\text { p-value - comparison } \\
\text { of } 2 \text { counts }\end{array}$} \\
\hline I vs II & 0.001 & 1.0 & 1.0 & & & & & 0.05 & 0.3 & 0.75 \\
\hline I vs III & 0.003 & 1.0 & 0.73 & & & & & 0.15 & 0.7 & 0.65 \\
\hline II vs III & 0.75 & 1.0 & 0.48 & & & & & 0.3 & 0.7 & 0.9 \\
\hline
\end{tabular}

Table II

The incidence of C. albicans in different body sites of women during one visit (60 samples)

\begin{tabular}{|l|c|}
\hline \multicolumn{1}{|c|}{ Body site } & Number of C. albicans samples (\%) \\
\hline oral cavity & $6(10.0)$ \\
\hline anus & $2(3.3)$ \\
\hline vagina & $1(1.6)$ \\
\hline anus - oral cavity & $10(16.7)$ \\
\hline vagina - oral cavity & $9(15.0)$ \\
\hline vagina - anus & $4(6.7)$ \\
\hline vagina - oral cavity - anus & $28(46.7)$ \\
\hline
\end{tabular}

study because of their single colonies in the culture in comparison with C. albicans. From 4 women 2 morphotypes of C. albicans in vaginal sample were isolated, whereas 3 (15\%) women experienced recurrent VC (J.A,
P.I, S.I.); the remaining ones were colonized without symptoms of vaginal infection in the 2 follow-up visits.

C.albicans strains were classified to 6 biotypes - Fig. 1. The most common was biotype C (58.8\%) which was found in 14 (70.0\%) of women, at the infection site (vagina I) and colonized body sites throughout the study time. C. albicans strains classified to biotype $\mathrm{Z}$ (described by the authors of the study) exhibited the activity three (esterase C4, valine arylamidase, $\mathrm{N}$-acetyl-beta-glucosaminidase) of five enzymes used for biotyping. Those strains were isolated only from vagina of 3 different women (B.Ka, K.D, W.Z.) during follow-up visits after vaginal candidiasis treatment. Each strain of biotype " $Z$ " presented a different genetic pattern, not observed in other body sites of these women.

Based on the average similarity coefficient $\left(\mathrm{S}_{\mathrm{AB}}=0.80\right)$ calculated for all studied C. albicans strains 31 genotypes

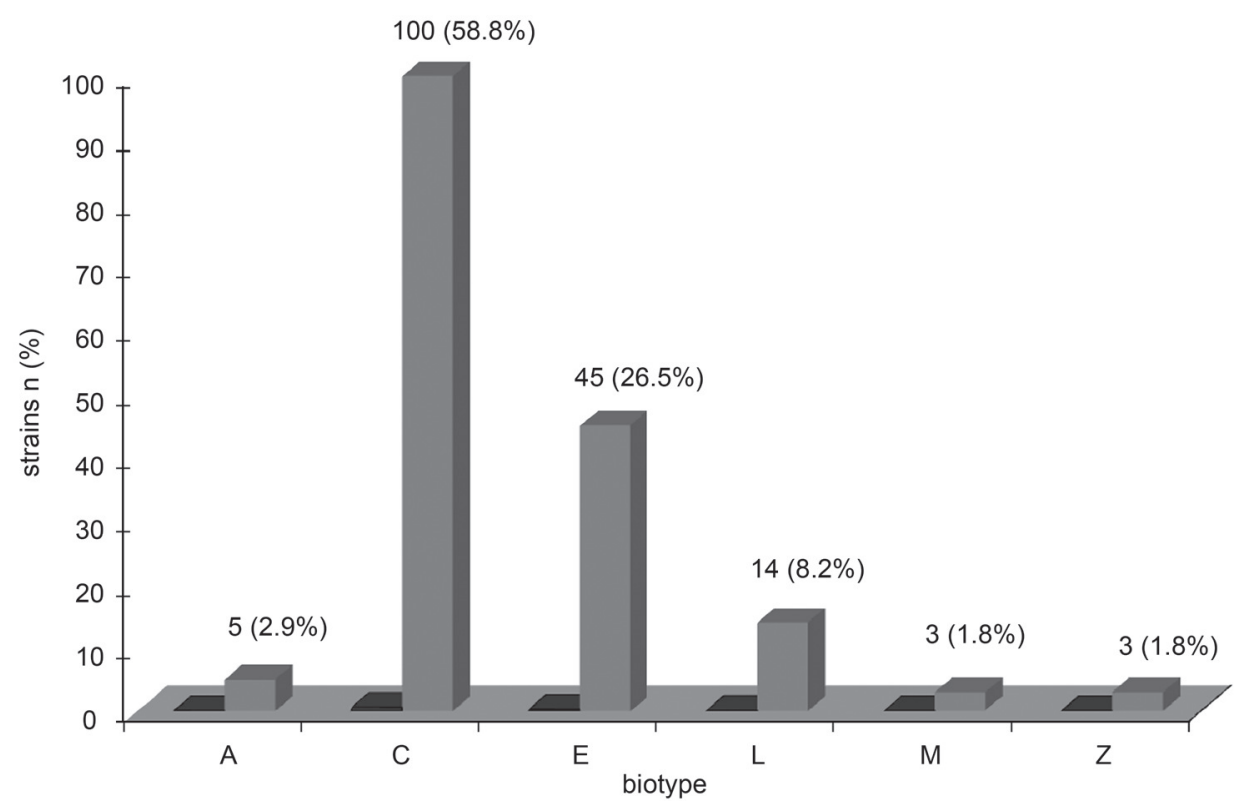

Fig. 1. Biotypes of C. albicans strains isolated from women with vaginal candidiasis 
Table III

The genotypes and biotypes of C. albicans strains isolated from vagina, oral cavity and anus of women with vaginal candidiasis during three consecutive visits

\begin{tabular}{|c|c|c|c|c|c|c|c|c|c|c|c|}
\hline \multirow[b]{2}{*}{ No. } & \multirow[b]{2}{*}{ Patient } & \multirow{2}{*}{$\begin{array}{c}\text { Age } \\
\text { (years) }\end{array}$} & \multicolumn{3}{|c|}{ VISIT I (before treatment) } & \multicolumn{3}{|c|}{ VISIT II (follow-up visit) } & \multicolumn{3}{|c|}{ VISIT III (follow-up visit) } \\
\hline & & & vagina & $\begin{array}{c}\text { oral } \\
\text { cavity }\end{array}$ & anus & vagina & $\begin{array}{c}\text { oral } \\
\text { cavity }\end{array}$ & anus & vagina & $\begin{array}{c}\text { oral } \\
\text { cavity }\end{array}$ & anus \\
\hline 1. & B.K. & 36 & $\begin{array}{l}\text { 27. } \mathrm{E} \\
\text { 27. } \mathrm{E}\end{array}$ & 27. $\mathrm{E}$ & 27. $E^{*}$ & & & 27. $\mathrm{E}$ & & 27. E & \\
\hline 2. & B.Ka. & 23 & u13. E & & & 7. Z & & 22. $\mathrm{E}$ & 22. $\mathrm{E}^{*}$ & & 22. $\mathrm{E}$ \\
\hline 3. & B.M. & 44 & 7. $\mathrm{E}$ & 12. $\mathrm{M}$ & 12. M & & 13. E & 13. $\mathrm{C}$ & & 12. $\mathrm{E}$ & 12. $\mathrm{E}$ \\
\hline 4. & F.A. & 25 & 16. E & u20. L & 14. $C^{*}$ & & 6. $\mathrm{C}$ & & 13. E & 1. $\mathrm{L}$ & \\
\hline 5. & F.U. & 36 & $\begin{array}{l}\text { 17. } \mathrm{C} \\
\text { 17. } \mathrm{E}\end{array}$ & 17. $\mathrm{C}$ & & & 17. $\mathrm{C}$ & 14. C & & 7. C & \\
\hline 6. & G.D. & 29 & 20. $\mathrm{C}$ & $\begin{array}{l}\text { u8. E } \\
\text { 21. } \mathrm{C} \\
\text { 23. } \mathrm{E}\end{array}$ & 18. C & & 21. E & 18. C & & $\begin{array}{l}\text { u11. E } \\
26 . \mathrm{E}\end{array}$ & $\begin{array}{l}\text { 18. C } \\
\text { 23. } \mathrm{E}\end{array}$ \\
\hline 7. & J.A. $^{\mathrm{R}}$ & 32 & 17. $\mathrm{C}$ & & u7. C & 17. C & 4. $C^{*}$ & 4. $\mathrm{C}$ & 17. C & 4. C & $\begin{array}{l}\text { 4. C } \\
\text { 4. C }\end{array}$ \\
\hline 8. & K.M. & 36 & 1.C & $\begin{array}{l}\text { 9. C } \\
\text { 10. L }\end{array}$ & 10. L & 10. E & 9. A & 11. C & 9. $\mathrm{C}$ & 9. C & $\begin{array}{l}\text { 9. C } \\
\text { 10. C }\end{array}$ \\
\hline 9. & K.D. & 38 & u16. C & 15. C & & & 15. C & & $\begin{array}{c}\text { 24. C } \\
\text { u15. Z }\end{array}$ & 25. $C^{*}$ & 24. C \\
\hline 10. & K.I. & 31 & $\begin{array}{l}\text { 29. C } \\
\text { u18. C }\end{array}$ & $\begin{array}{l}\text { u9. C } \\
\text { u19. C }\end{array}$ & 29. $\mathrm{E}^{*}$ & 28. E & u17. C & 28. L & 28. E & 30. C & \\
\hline 11. & M.A. & 23 & 10. $\mathrm{E}$ & $\begin{array}{l}\text { 1. } \mathrm{C} \\
\text { 7. } \mathrm{E}\end{array}$ & 11. E & & 1. $\mathrm{E}$ & 11. E & & 8. $\mathrm{E}$ & 1. $\mathrm{E}$ \\
\hline 12. & M.K. & 26 & $\begin{array}{l}\text { u3. C } \\
\text { u5. A }\end{array}$ & u4. A & 4. E & & 4. A & $\begin{array}{l}\text { 4. A } \\
\text { 4. } \mathrm{L}\end{array}$ & & 4. $\mathrm{M}$ & \\
\hline 13. & P.I. ${ }^{\mathrm{R}}$ & 42 & 6. L & $\begin{array}{l}\text { 8. C } \\
\text { 16. C }\end{array}$ & & 6. L & $\begin{array}{l}\text { u2. C } \\
\text { u6. L }\end{array}$ & $\begin{array}{l}\text { 15. L } \\
\text { 16. C }\end{array}$ & 6. $\mathrm{L}^{*}$ & $\begin{array}{l}\text { 13.C } \\
\text { 16. L }\end{array}$ & 16. C \\
\hline 14. & R.A. & 29 & 1. C & 2. $\mathrm{C}$ & 2. C & & $\begin{array}{l}\text { 2. C } \\
\text { u1. C }\end{array}$ & 2. $\mathrm{C}$ & & 2. C & 2. $\mathrm{C}$ \\
\hline 15. & R.Ag. & 26 & u12. C & 17. C & 17. C & 17. C & 17. C & 17. C & 17. C & 17. C & \\
\hline 16. & S.I. ${ }^{\mathrm{R}}$ & 32 & 31. $\mathrm{E}$ & 31. C & 31. E & 31. E & 31. C & 31. E & 31. E & 31. C & 31. E \\
\hline 17. & S.A. & 36 & 30. C & 23. $\mathrm{C}$ & 30. C & & & 30. C & 22. C & & 3. C \\
\hline 18. & S.Z. & 41 & 5. C & 26. $\mathrm{C}$ & 26. L & 4. $\mathrm{C}$ & 5. C & & & 12. C & \\
\hline 19. & W.Z. & 44 & 19. C & 17. $C^{*}$ & & 19. C & 17. $\mathrm{E}$ & & 20. Z & 17. $\mathrm{E}$ & 22. $\mathrm{C}$ \\
\hline 20. & Z.E. & 21 & u10. C & 19. C & 19. $C^{*}$ & 19. C & 19. C & 19. C & 3. $\mathrm{C}$ & 3. C & $\begin{array}{l}\text { 19. C } \\
\text { u14. C }\end{array}$ \\
\hline
\end{tabular}

${ }^{\mathrm{R}}$ - women with recurrent vaginal candidiasis; ${ }^{\star}$ two C. albicans isolates with different morphotypes presented the same genotype Numbers denote genotype, capital letters denote biotype, $\mathrm{u}-$ unrelated genetic patterns

were revealed that grouped strains being identical $\left(\mathrm{S}_{\mathrm{AB}}=1\right)$ and genetically related isolates $\left(\mathrm{S}_{\mathrm{AB}}=0.80-0.99\right)$. The 20 unrelated isolates ('u') with unique patterns $\left(\mathrm{S}_{\mathrm{AB}}<0.80\right)$ were found (Table III). C. albicans strains from each woman were assigned to $1-5$ genotypes. Usually a single genotype of C.albicans was found in oral cavity, anus and vagina, in $40 \%, 65 \%$ and $55 \%$ of patients, respectively. The occurrence of two genotypes of C. albicans at a single body site was observed in $30-35 \%$ of the women, while the three genotypes in
5-20\%. There were no statistically significant differences in the occurrence of one, two or three genotypes of C. albicans at a single body site. Based on average similarity coefficient between pairs of $C$. albicans isolates revealed that pairs of vaginal and anal isolates were more similar $\left(\mathrm{S}_{\mathrm{AB}}=0.80 \pm 0.05\right)$ than other pairs of isolates. The similarity of vaginal/oral and anal/oral pairs of strains was $\mathrm{S}_{\mathrm{AB}}=0.74 \pm 0.04$ and $\mathrm{S}_{\mathrm{AB}}=0.75 \pm 0.01$, respectively.

C. albicans strains belonging to the same genotype and biotype were isolated concomitantly from 2 
or 3 body sites, from 13 and 4 women, respectively as well as 2 or 3 times at time intervals from the same body site, from 12 and 7 women, respectively. The same genotypes were found in epidemiologically and geographically unrelated women; genotype 17 (18 isolates) was observed in the case of 4 (F.U, J.A, R.Ag, W.Z.) and genotype 4 (12 isolates) for 3 women (J.A, M.K, S.Z.). The same genotypes were found in the vagina (J.A, P.I.) and in oral/anal samples (S.I.) of women with recurrent $\mathrm{VC}$ as well as in vagina (W.Z.) or in oral/anal sites of women without symptoms of VC (B.K, F.U, S.A, S.Z.).

The C.albicans strains of different genotypes and biotypes were isolated concomitantly from different body sites and repeatedly at time intervals from the same body site (K.I, K.M, S.Z, F.A.). Some women had a unique genetic pattern of strains at the site of infection (vagina/visit I): u13 (B.Ka.); u16 (K.D.); u18 (K.I.); u3 i u5 (M.K.); u12 (R.Ag.); u10 (Z.E.) that were characteristic of the site of infection. Unique genetic patterns of strain also occurred at colonized body sites.

The results of the study show that C.albicans is still the most isolated yeast species regardless of body site and sampling time. Most of the strains of this species were eliminated from the vagina after antifungal therapy with nystatin but some of them persisted a few months in the vagina of women with and without VC symptoms. Similarly, the results of other authors suggest that topical antifungal agents resolve the symptoms of VC in the majority of women, but do not completely eliminate yeast from the vagina (Lanchares and Hernández, 2000; Mercure et al., 1993; Sobel, 2007; Sobel, 2003; Sobel, 1996; Soll et al., 1991; Vazquez et al., 1994). Unfortunately, some women are still colonized after VC treatment, yet others have a relapse of VC (Sobel, 1985). Yeast-positive cultures appeared 30 days or 3 months after the completion of therapy of VC, in 15-20\% of women in Odds findings (1982) and in $20-25 \%$ of women according to Sobel (2007), respectively. In our study up to $50 \%$ of women had a yeastpositive vaginal culture 2 months after treatment and re-colonization increased after 4 months. Some authors intensify the research concerning the yeast colonization of the other body sites to help in the treatment of VC and to improve the satisfactory efficacy in the management of VC in clinical practice. The origin of VC is multifactorial and may be associated not only with incomplete elimination of yeasts from the vaginal epithelium but also with re-colonization from other host body sites or from the external environment. Although it was commonly believed that the proximity of the rectum colonized by yeast is a predisposing factor for $\mathrm{VC}$, our report exhibited more frequent oral than anal colonization before and after antifungal therapy of VC. Similarly to our results, Soll at al. (1991) found a higher percentage of yeast in the oral cavity (56\%) than in the anorectal region (24\%) and the frequent relationship of carriage between pairs of oral-anal and oral-vaginal isolates. The mentioned above data together with Sobel's results $(2007,1985)$ suggested that the importance of rectal isolates is overestimated in the pathogenesis of VC. Based on typing systems we tried to answer the question of yeast source involved in VC. Biotyping poorly typed C.albicans isolates because up to $90 \%$ of women with VC were colonized by strains belonging to biotype $\mathrm{C}$ before and after antifungal therapy. Comparative analysis with other studies (Brajer et al., 2005; Kurnatowska and Kurnatowski, 1998) was difficult because of geographically different samples, not related patients or application of different kinds of classification. The results of molecular typing, by means of optimized RAPD technique, revealed greater diversity of C. albicans types in different body sites of the same patient in comparison with the results of biotyping.

The DNA-based typing methods of C.albicans strains revealed the highest genetic similarity between pairs of vaginal and anal strains in comparison with other pairs of isolates. The results of genotyping seem to be consistent with the results of earlier studies conducted by Mercure et al. (1993) and Mendling et al. (2000). The former authors suggested the anal isolates could be a reason for VC because their genetic similarity with vaginal isolates was found in $68 \%$ of episodes, the latter demonstrated that different body sites including oral cavity might be the endogenous source of yeast. The results of the present genotyping study do not exclude both points of view.

Colonization of one body site by the same biotype/ genotype over an extended time can suggest endogenous re-infection or vaginal relapse. The hypotheses seem to be more acceptable for women with recurrent VC (J.A, P.I, S.I) and they are consistent with the results of Vazquez et al. (1994). The studies conducted by other authors (Lockhart et al. 1996; Maffei et al., 1997) in women with recurrent VC also suggested the persistence of the single yeast genotype within vagina during consecutive episodes of VC. It can be associated with incomplete elimination of the specific genotype after topical treatment (El-Din et al., 2001; Stein et al., 1991). In the light of Fischer's (2012) data patients frequently do not have recurrent symptoms of VC but their disease is chronic as a result of oestrogen-related yeast persistence (Fischer, 2012).

The variability of biotypes/genotypes within one body site and within one woman over an extended time could be the result of topical antifungal therapy as well as may suggest the acquisition of new isolates by the host. The emergence of biotype $\mathrm{Z}$ after antifungal therapy in the control visits can be the result of selection of C. albicans phenotypes, while the occurrence of unique genetic patterns can suggest the acquisition of the 
infecting isolates from an exogenous source. The occurrence of multiple genotypes and biotypes of C. albicans at the woman's body sites suggests a dynamic process of woman colonization by yeast, including the exchange of strains/genotypes within body sites. The findings are consistent with the results of Stein et al. (1991), who observed the exchange of strains in 4 of 7 women with VC. The study conducted by Samaranyake at al. (2003) on oral isolates also confirms the dynamic colonization of a body site by multiple genotypes.

No specific relationships were found between genotype/biotype/body site and clinical symptoms. The data are consistent with the results of Lian et al. (2004); Mercure et al. (1993) and Xu et al.(1999). Thus, these results rather suggest a circulation of the multiple genotypes in the population and the need to seek other factors responsible for the symptoms of infection. The factors can be associated with the host immune response and yeast adaptation to specific environmental conditions, i.e. with the adhesion to host tissues or with susceptibility to drugs (work in progress).

In summary, the results of our study indicate that VC can be both endogenous and exogenous in origin. The occurrence of an identical genotype and biotype at the site of infection and/or colonization over several months confirmed the endogenous relapse, transmission of the strains between body sites and the possibility of endogenous re-infection. The diversity of genotypes at the body sites of the same individual over a few months confirmed the periodic exchange of strains and their acquisition from an exogenous source. Do these processes appear occasionally or are they dependent on some other factors? This is a question for further research.

\section{Literature}

al-Rawi N. and K. Kavanagh. 1999. Characterisation of yeasts implicated in vulvovaginal candidosis in Irish women. Br. J. Biomed. Sci. 56: 99-104.

Amouri I.H. Sellami, N. Borji, S. Abbes, A. Sellami, F. Cheikhrouhou, L. Maazoun, S. Khaled, S. Khrouf, Y. Boujelben and others. 2010. Epidemiological survey of vulvovaginal candidosis in Sfax, Tunisia. Mycoses 54: 499-505.

Brajer B., H. Batura-Gabryel, C. Łukaszuk, M. Mnichowska, E. Krajewska-Kulak and S. Giedrys-Kalemba. 2005. Biotypes of Candida albicans isolated from the upper respiratory tract from healthy people living in three geographical regions of Poland (in Polish). Mikol. Lek. 12: 109-113.

Chong P.P., Y.L. Lee, B.C. Tan and K.P. Ng. 2003. Genetic relatedness of Candida strains isolated from women with vaginal candidiasis in Malaysia. J. Med. Microbiol. 52: 657-666.

Cobb B.D. and J.M. Clarkson. 1994. A simple procedure for optimising the polymerase chain reaction (PCR) using modified Taguchi methods. Nucleic Acids Res. 22: 3801-3805.

Cobb B.D. and J.M. Clarkson. 1992. Optimization of RAPD fingerprinting, pp. 93-102. In: Micheli M.R. and R. Bova (eds).
Fingerprinting methods based on arbitrarily primed PCR. SpringerVerlag, Berlin Heidelberg New York.

Darce Bello M., A. Gonzalez, C. Barnabe and G. Larrouy. 2002. First characterization of Candida albicans by random amplified polymorphic DNA method in Nicaragua and comparison of the diagnosis methods for vaginal candidiasis in Nicaraguan women. Mem. Inst. Oswaldo Cruz. 97: 985-989.

El-Din S.S., M.T. Reynolds, H.R. Ashbee, R.C. Barton and E.G. Evans. 2001. An investigation into the pathogenesis of vulvovaginal candidosis. Sex Transm. Infect. 77: 179-183.

Eschenbach D.A. 2008. Chronic vulvovaginal candidiasis. N. Engl. J. Med. 351: 851-852.

Fischer G. 2012. Chronic vulvovaginal candidiasis: What we know and what we have yet to learn. Australasian J. Dermatol. 53: 247-254. Ge S.H, J. Xie, J. Xu, J. Li, D.M. Li, L.L. Zong, Y.C. Zheng and F.Y. Bai. 2012. Prevalence of specific and phylogenetically closely relat ed genotypes in the population of Candida albicans associated with genital candidiasis in China. Fungal Genet. Biol. 49: 86-93.

Hurley R. and J. DeLouvois. 1979. Candida vaginitis. Postgrad. Med. J. 55: 645-647.

Ilkit M. and A.B. Guzel. 2011. The epidemiology, pathogenesis, and diagnosis of vulvovaginal candidosis: a mycological perspective. Crit. Rev. Microbiol. 37: 250-261. Epub 2011, May 20 Review. Kurnatowska A.J. and P. Kurnatowski. 1998. Biotypes of fungi isolated from patients with oral cavity diseases. Mikol. Lek. 5: 213-217. Lanchares J.L. and M.L. Hernández. 2000. Recurrent vaginal candidosis changes in etiopathogenical patterns. Int. J. Gynaecol. Obstet. 71 Suppl 1: 29-35.

Li J., S.R. Fan, X.P. Liu, D.M. Li, Z.H. Nie, F. Li, H. Lin, W.M. Huang, L.L. Zong, J.G. Jin and others. 2008. Biased genotype distributions of Candida albicans strains associated with vulvovaginal candidosis and candidal balanoposthitis in China. Clin. Infect. Dis. 47: 1119-1125.

Lian C., J. Zhao, Z. Zhang and W. Liu. 2004. Genotype of Candida species associated with different conditions of vulvovaginal candidosis. Mycoses 47: 495-502.

Lockhart S.R., B.D. Reed, C.L. Pierson and D.R. Soll. 1996. Most frequent scenario for recurrent Candida vaginitis is strain maintenance with "substrain shuffling": demonstration by sequential DNA fingerprinting with probes Ca3, C1 and CARE2. J. Clin. Microbiol. 34: 767-777.

Maffei C.M., C.R. Paula, T.S. Mazzocato and S. Franceschini. 1997. Phenotype and genotype of Candida albicans strains isolated from pregnant women with recurrent vaginitis. Mycopathologia 137: 87-94.

Mahmoudi Rad M., S. Zafarghandi, B. Abbasabadi and M. Tavallaee. 2011. The epidemiology of Candida species associated with vulvovaginal candidiasis in an Iranian patient population. Eur. J. Obstet. Gynecol. Reprod. Biol. 155: 199-203.

Mendling W., M.P. De Andrade, J. Gutschmidt, R. Gantenberg, W. Presber and G. Schőnian. 2000. Strain specificity of yeasts isolated from different locations of women suffering from vaginal candidosis, and their partners. Mycoses 43: 387-392.

Mercure S., S. Poirier, G. Lemay, P. Auger, S. Montplaisir and L. De Repentigny. 1993. Application of biotyping and DNA typing of Candida albicans to the epidemiology of recurrent vulvovaginal candidiasis. J. Infect. Dis. 168: 502-507.

Odds F.C. 1982. Genital candidosis. Clin. Exp. Dermatol. 7: 345.

Ringdahl E.N. 2000. Treatment of recurrent vulvovaginal candidiasis. Am. Fam. Physician. 61: 3306-3312.

Samaranayake Y.H., L.P. Samaranayake, J.Y. Yau, A.N. Ellepola, S. Anil and K.W. Yeung. 2003. Adhesion and cell-surface-hydrophobicity of sequentially isolated genetic isotypes of Candida albicans in an HIV-infected Southern Chinese cohort. Mycoses 46: 375-383. 
Schmid J., M. Rotman, B. Reed, C.L. Pierson and D.R. Soll. 1993. Genetic similarity of Candida albicans strains from vaginitis patients and their partners. J. Clin. Microbiol. 31: 39-46.

Schmid J., E. Voss and D.R. Soll. 1990. Computer-assisted methods for assessing strain relatedness in Candida albicans by fingerprinting with the moderately repetitive sequence Ca3. J. Clin. Microbiol. 28: 1236-1243.

Sobel J.D. 2007. Vulvovaginal candidosis. Lancet 369: 1961-1971. Sobel J.D. 2003. Management of patients with recurrent vulvovaginal candidiasis. Drugs 63: 1059-1066.

Sobel J.D. 1996. Candida vulvovaginitis. Semin. Dermatol. 15: 17-28. Sobel J.D. 1993. Candidal vulvovaginitis. Clin. Obstet. Gynecol. 36: 153-165.

Sobel J.D. 1990. Vaginal infections in adult women. Med. Clin. North. Am. 74: 1573-1602.

Sobel J.D. 1985. Epidemiology and pathogenesis of recurrent vulvovaginal candidiasis. Am. J. Obstet. Gynecol. 152: 924-935.

Soll D. 2000. The ins and outs of DNA fingerprinting the infectious fungi. Clin. Microbiol. Rev. 13: 332-370.
Soll D.R., R. Galask, J. Schmid, C. Hanna, K. Mac and B. Morrow. 1991. Genetic dissimilarity of commensal strains of Candida spp. carried in different anatomical locations of the same healthy women. J. Clin. Microbiol. 29: 1702-1710.

Stein G.E., V.L. Sheridan, B.B. Magee and P.T. Magee. 1991. Use of rDNA restriction fragment length polymorphisms to differentiate strains of Candida albicans in women with vulvovaginal candidiasis. Diagn. Microbiol. Infect. Dis. 14: 459-464.

Vazquez J.A., J.D. Sobel, R. Demitriou, J. Vaishampayan, M. Lynch and M.J. Zervos. 1994. Kariotyping of Candida albicans isolates obtained longitudinally in women with recurrent vulvovaginal candidiasis. J. Infect. Dis. 170: 1566-569.

Williamson M.I., L.P. Samaranayake and T.W. MacFarlane. 1986. Biotypes of oral Candida albicans and Candida tropicalis isolates. J. Med. Vet. Mycol. 24: 81-84.

Xu J., C.M. Boyd, E. Livingston, W. Meyer, J.F. Madden and T.G. Mitchell. 1999. Species and genotypic diversities and similarities of pathogenic yeasts colonizing women. J. Clin. Microbiol. 37: 3835-3843. 